\title{
Entanglement entropy, conformal invariance and extrinsic geometry
}

\author{
Sergey N. Solodukhin 1 \\ Laboratoire de Mathématiques et Physique Théorique CNRS-UMR 6083, \\ Université de Tours, Parc de Grandmont, 37200 Tours, France
}

\begin{abstract}
We use the conformal invariance and the holographic correspondence to fully specify the dependence of entanglement entropy on the extrinsic geometry of the $2 \mathrm{~d}$ surface $\Sigma$ that separates two subsystems of quantum strongly coupled $\mathcal{N}=4 S U(N)$ superconformal gauge theory. We extend this result and calculate entanglement entropy of a generic $4 \mathrm{~d}$ conformal field theory. As a byproduct, we obtain a closed-form expression for the entanglement entropy in flat space-time when $\Sigma$ is sphere $S_{2}$ and when $\Sigma$ is two-dimensional cylinder. The contribution of the type A conformal anomaly to entanglement entropy is always determined by topology of surface $\Sigma$ while the dependence of the entropy on the extrinsic geometry of $\Sigma$ is due to the type B conformal anomaly.
\end{abstract}

\footnotetext{
${ }^{1}$ solodukh@lmpt.univ-tours.fr
} 


\section{Introduction}

Entanglement entropy is a well-defined measure of the quantum correlations between two subsystems separated by a surface $\Sigma$. Since the correlations are short-range the entropy is essentially determined by geometry, both intrinsic and as an embedding into a larger space-time, of the surface $\Sigma$. The recent studies revealed the important role the entanglement entropy plays in the physics of black holes and in the quantum field models [1]-17]. An important element of the present understanding is the holographic interpretation of entanglement entropy [18], [19]. This interpretation suggests a purely geometric way of computing the entropy of a strongly coupled conformal field theory [20]-[24].

In this note we give a derivation of entanglement entropy of a generic four-dimensional conformal field theory based on the conformal properties of the theory. Our approach is partially motivated by work of Dowker [25]. In particular, we suggest that, in the large $\mathrm{N}$ limit, the entanglement entropy calculated in a four-dimensional $\mathcal{N}=4 S U(N)$ superconformal gauge theory, the quantum field theory counterpart in the holographic duality, is given by expression

$$
S_{S C}=\frac{A(\Sigma)}{4 \pi \epsilon^{2}} N^{2}-\frac{N^{2}}{24 \pi} \int_{\Sigma}\left(3 R_{a a}-2 R-\frac{3}{2} k_{a} k_{a}\right) \ln \epsilon+s_{S C}(g),
$$

where $A(\Sigma)$ is area of $\Sigma, R$ is the Ricci scalar of four-dimensional metric $g_{\mu \nu}, R_{a a}=$ $\sum_{i=1}^{2} R_{\mu \nu} n_{a}^{\mu} n_{a}^{\nu}$ is projection of the Ricci tensor onto the subspace orthogonal to surface $\Sigma$, $n_{a}^{\mu}, a=1,2$ is a pair of unite vectors orthogonal to the surface $\Sigma$. $k_{a}$ is the trace of the

second fundamental form $k_{\mu \nu}^{a}=-\gamma_{\mu}^{\alpha} \gamma_{\nu}^{\beta} \nabla_{\alpha} n_{\beta}^{a}$, where $\gamma_{\mu \nu}=g_{\mu \nu}-n_{\mu}^{a} n_{\nu}^{a}$ is the induced metric of $\Sigma$. $\epsilon$ is an UV cut-off needed for regularization of the theory on curved background.

The logarithmic term in (1.1) is invariant under generic conformal transformations, $g_{\mu \nu} \rightarrow e^{-2 \omega} g_{\mu \nu}$, in particular under those with non-vanishing normal derivatives of $\omega$ on $\Sigma$. The term $k^{a} k^{a}$ in (1.1) takes care of the invariance under these particular transformations.

The function $s_{S C}(g)$ in (1.1) is the UV finite part of the entropy. Under global rescaling of the metric, $g_{\mu \nu} \rightarrow \lambda^{2} g_{\mu \nu}$, this function changes as

$$
s_{S C}\left(\lambda^{2} g\right)=s_{S C}(g)+\frac{N^{2}}{24 \pi} \int_{\Sigma}\left(3 R_{a a}-2 R-\frac{3}{2} k_{a} k_{a}\right) \ln \lambda .
$$

Thus, the logarithmic term in (1.1) and (1.2) is due to the integrated conformal anomaly. This aspect will be clarified below. The property (1.2) is particularly useful if the configuration of surface $\Sigma$ in $4 \mathrm{~d}$ space-time is characterized by only one dimensionful parameter, $a$. Then, rescaling the $4 \mathrm{~d}$ metric and using (1.2) one can determine the dependence of the entropy on this parameter. The logarithmic term in entanglement entropy of a black hole was obtained in [6] and further studied in [7, [9], [11, [10].

The expression (1.1) is general in that it gives the entropy for all possible choices of surface $\Sigma$. In particular, $\Sigma$ can be a black hole horizon. The extrinsic curvature $k^{a}, a=1,2$ vanishes in this case. The other possible choice of $\Sigma$ is any compact surface in curved space-time. Of a particular interest is a surface in flat space-time. Then, the logarithmic contribution to the entropy is given entirely by the extrinsic curvature of $\Sigma$.

The entropy (1.1) has a holographic interpretation. In fact, the holographic origin of the logarithmic term was already verified in [19] in the no black hole case and in [22] for a 
black hole horizon. In both cases the extrinsic curvature of $\Sigma$ assumed to be zerd2. Thus, a goal of this note is to single out the important contribution of the extrinsic curvature to the logarithmic term in (1.1). The holographic interpretation of entanglement entropy was questioned recently in [27]. Below we comment on this.

That entanglement entropy may depend on the extrinsic curvature of $\Sigma$ was earlier suggested in [12] based on the heat kernel analysis of Dowker [25].

\section{Replica method, singular surface and the confor- mal structure of effective action}

The most efficient method to compute entanglement entropy is to introduce at the surface $\Sigma$ a conical singularity of small angle deficit $\delta=2 \pi(1-\alpha)$, compute the effective action $W\left(E^{\alpha}\right)$ on a manifold $E^{\alpha}$ with a singular surface and then apply the formula

$$
S=\left.\left(\alpha \partial_{\alpha}-1\right)\right|_{\alpha=1} W\left(E^{\alpha}\right)
$$

and obtain the entanglement entropy. Explanation of this method is available in papers [4], [15].

In this note we are interested in entanglement entropy of a four-dimensional conformal field theory. The effective action then has a general structure

$$
W_{\mathrm{CFT}}\left(E^{\alpha}\right)=\frac{a_{0}}{\epsilon^{4}}+\frac{a_{1}}{\epsilon^{2}}+a_{2} \ln \epsilon+w\left(g^{(\alpha)}\right) .
$$

The terms $a_{0}$ and $a_{1}$, representing the polynomial UV divergences, are not universal while the term $a_{2}$ is universal and is determined by the integrated conformal anomaly. $w\left(g^{(\alpha)}\right)$ is the UV finite part of the effective action. Under a global rescaling of the $4 \mathrm{~d}$ metric on $E^{\alpha}, g^{(\alpha)} \rightarrow \lambda^{2} g^{(\alpha)}$, one has

$$
w\left(\lambda^{2} g^{(\alpha)}\right)=w\left(g^{(\alpha)}\right)-a_{2} \ln \lambda .
$$

It is proved (see [33] and references therein) on a rather general grounds that for a generic manifold and a generic conformal field the corresponding coefficient $a_{2}$ is conformal invariant, $a_{2}\left[e^{-2 \omega} g\right]=a_{2}[g]$. This is an important property that plays a key role in our consideration.

On $4 \mathrm{~d}$ manifold with a singular surface $\Sigma$ the coefficients $a_{1}$ and $a_{2}$ have both the bulk part and the surface part. To first order in $(1-\alpha)$ one finds that

$$
\begin{aligned}
& a_{1}\left(E^{\alpha}\right)=\alpha a_{1}^{\text {bulk }}(E)+(1-\alpha) a_{1}^{\Sigma}+O(1-\alpha)^{2}, \\
& a_{2}\left(E^{\alpha}\right)=\alpha a_{2}^{\text {bulk }}(E)+(1-\alpha) a_{2}^{\Sigma}+O(1-\alpha)^{2},
\end{aligned}
$$

where

$$
a_{1}^{\mathrm{bulk}}(E)=C \int_{E} R, \quad a_{1}^{\Sigma}=4 \pi C \int_{\Sigma} 1
$$

with constant $C$ depending on the UV regularization scheme. In this note we use normalization $C=\frac{1}{16 \pi^{2}}$. Coefficients $a_{2}^{\text {bulk }}(E)$ and $a_{2}(\Sigma)$ are respectively the integrated bulk

\footnotetext{
${ }^{2}$ Black hole horizon is a fixed point of abelian isometry. The extrinsic curvature of horizon, thus, vanishes.
} 
and surface conformal anomalies. Under a conformal transformation $g \rightarrow e^{-2 \omega} g$ one has that

$$
a_{2}^{\text {bulk }}\left(e^{-2 \omega} g\right)=a_{2}^{\text {bulk }}(g) \text { and } a_{2}^{\Sigma}\left(e^{-2 \omega} g\right)=a_{2}^{\Sigma}(g)
$$

Applying the formula (2.1) one obtains entanglement entropy

$$
\begin{aligned}
& S=\frac{a_{1}^{\Sigma}}{\epsilon^{2}}+a_{2}^{\Sigma} \ln \epsilon+s(g), \\
& s\left(\lambda^{2} g\right)=s(g)-a_{2}^{\Sigma} \ln \lambda .
\end{aligned}
$$

In four dimensions the bulk conformal anomaly is a combination of two terms, the topological Euler term and the square of the Weyl tensor,

$$
\begin{aligned}
& a_{2}^{\text {bulk }}=A E_{(4)}+B I_{(4)}, \\
& E_{(4)}=\frac{1}{64} \int_{E}\left(R_{\alpha \beta \mu \nu} R^{\alpha \beta \mu \nu}-4 R_{\mu \nu} R^{\mu \nu}+R^{2}\right), \\
& I_{(4)}=-\frac{1}{64} \int_{E}\left(R_{\alpha \beta \mu \nu} R^{\alpha \beta \mu \nu}-2 R_{\mu \nu} R^{\mu \nu}+\frac{1}{3} R^{2}\right) .
\end{aligned}
$$

These are respectively the conformal anomalies of type A and B. The surface contribution to the conformal anomaly can be calculated directly by, for example, the heat kernel method as in [28]. The direct computation although straightforward is technically involved. One has however a short cut: there is a precise balance, observed in [6] and [8, between the bulk and surface anomalies, this balance is such that to first order in $(1-\alpha)$ one can take $a_{2}\left(E^{\alpha}\right)=a_{2}^{\text {bulk }}\left(E^{\alpha}\right)+O(1-\alpha)^{2}$ and use for the Riemann tensor of $E^{\alpha}$ the representation as a sum of regular and singular (proportional to a delta-function concentrated on surface $\Sigma$ ) parts. The precise expressions are given in [8], [26]. This representation, however, is obtained under the assumption that the surface $\Sigma$ has the extrinsic curvature vanishing. Under this assumption one finds that [8], [26]

$$
\begin{aligned}
& a_{2}\left(E^{\alpha}\right)=\alpha a_{2}^{\text {bulk }}(E)+(1-\alpha) a_{2}^{\Sigma}+O(1-\alpha)^{2}, \\
& a_{2}^{\Sigma}=A a_{A}^{\Sigma}+B a_{B}^{\Sigma}, \\
& a_{A}^{\Sigma}=\frac{\pi}{8} \int_{\Sigma}\left(R_{a b a b}-2 R_{a a}+R\right), \\
& a_{B}^{\Sigma}=-\frac{\pi}{8} \int_{\Sigma}\left(R_{a b a b}-R_{a a}+\frac{1}{3} R\right),
\end{aligned}
$$

where $R_{a b a b}=R_{\alpha \beta \mu \nu} n_{a}^{\alpha} n_{b}^{\beta} n_{a}^{\mu} n_{b}^{\nu}, R_{a a}=R_{\alpha \beta} n_{a}^{\alpha} n_{a}^{\beta}$.

Each surface term in (2.9) is invariant under a sub-class of conformal transformations, $g \rightarrow e^{-2 \omega} g$, such that the normal derivatives of $\omega$ vanish on surface $\Sigma$. The surface term due to the bulk Euler number is, moreover, a topological invariant: using the GaussCodazzi equation (A.5) and in the assumption of vanishing extrinsic curvature this term, as shown in [26], is proportional to the Euler number of the 2 d surface $\Sigma$,

$$
a_{A}^{\Sigma}=\frac{\pi}{8} \int_{\Sigma} R_{\Sigma}
$$

where $R_{\Sigma}$ is intrinsic curvature of $\Sigma$. 
As we discussed above the coefficient $a_{2}$ is conformal invariant and thus it should be invariant under arbitrary conformal transformations. The equation (2.9) is however invariant under a specific subclass of the conformal transformations, those with the normal derivatives vanishing on $\Sigma$. This is because the terms depending on the extrinsic geometry are neglected in (2.9). Such terms however become very important for the invariance of $a_{2}$ on manifold with a "squashed conical singularity" (in the terminology of Dowker [25]) that has a structure of the product of a conical metric and the surface $\Sigma$ scaled by an arbitrary conformal factor.

Thus, our goal in this section is to find a modification of (2.9) that would be invariant under a generic conformal transformation with the normal derivatives of $\omega$ non-vanishing on $\Sigma$. A generalization can be easily found with the help of formulae (A.4), (A.5) and (A.6) presented in Appendix.

The terms with the normal derivatives of $\omega$ in the conformal transformation of $a_{2}^{\Sigma}$ then can be cancelled by adding the quadratic combinations of extrinsic curvature, $\operatorname{tr} k^{2}$ and $k_{a} k_{a}$. We notice that the conformal covariant combination $\left(\operatorname{tr} k^{2}-\frac{1}{2} k_{a} k_{a}\right)$ always can be added with a numerical pre-factor, to be further specified. Additionally to the requirement of the conformal invariance we demand that the surface term in the anomaly determined by the Euler number to be topological invariant (2.10) even if the extrinsic curvature of $\Sigma$ is non-vanishing. This latter condition completely fixes the conformal covariant part in $a_{A}^{\Sigma}$. One then obtains

$$
\begin{aligned}
& a_{2}^{\Sigma}=A a_{A}^{\Sigma}+B a_{B}^{\Sigma}, \\
& a_{A}^{\Sigma}=\frac{\pi}{8} \int_{\Sigma}\left(R_{a b a b}-2 R_{a a}+R-\operatorname{tr} k^{2}+k_{a} k_{a}\right)=\frac{\pi}{8} \int_{\Sigma} R_{\Sigma}, \\
& a_{B}^{\Sigma}=-\frac{\pi}{8} \int_{\Sigma}\left(R_{a b a b}-R_{a a}+\frac{1}{3} R+\mu\left(\operatorname{tr} k^{2}-\frac{1}{2} k_{a} k_{a}\right)\right) .
\end{aligned}
$$

The method used in this section to determine $a_{2}^{\Sigma}$ is that of Dowker [25]. The value of $\mu$ can not be determined by the conformal invariance. In section 4 we use the holographic correspondence in order to specify $\mu 3$.

The surface anomalies have been recently studied in [27] and a non-conformal invariant form of the type A anomaly has been found. Although this issue requires a further analysis we note that, as equations (2.8) and (2.11) indicate, a simple form of the decomposition, like the one used in the cohomological analysis of [27], of the Riemann tensor on $E^{\alpha}$ on regular and singular parts is likely not valid when the extrinsic curvature of $\Sigma$ is non-vanishing.

\section{Entanglement entropy of $\mathcal{N}=4 S U(N)$ super Yang- Mills theory}

Provided the value of constant $\mu$ in (2.11) is determined (this will be done in section 4) the result (2.11) can be used to calculate the entanglement entropy of any conformal field theory in four dimensions. In this section we consider a particular conformal field theory that has a holographic dual description in terms of the gravity on anti-de Sitter space-time.

\footnotetext{
${ }^{3}$ We assume that $\mu$ does not depend on the field content of the theory.
} 
In the large $N$ limit the $\mathcal{N}=4 S U(N)$ superconformal gauge theory is characterized by the conformal anomaly, computed holographically in [29], that takes the form (2.8) with

$$
A=B=\frac{N^{2}}{\pi^{2}}
$$

On thus gets

$$
a_{2(S C)}^{\Sigma}=\frac{N^{2}}{24 \pi} \int_{\Sigma}\left(-3 R_{a a}+2 R+\frac{3}{2} k_{a} k_{a}-3(\mu+1)\left(\operatorname{tr} k^{2}-\frac{1}{2} k_{a} k_{a}\right)\right)
$$

for the surface term. The entanglement entropy of the superconformal gauge theory then has the form (2.7)

$$
S_{S C}=\frac{A(\Sigma)}{4 \pi \epsilon^{2}}+a_{2(S C)}^{\Sigma} \ln \epsilon+s_{S C}(g)
$$

As we show in the next section the holographic correspondence predicts value $\mu=-1$. With this value the entropy of the superconformal gauge theory is given by (1.1) as announced in the introduction.

\section{The holographic calculation}

In the holographic duality the quantum field theory is placed on a regularized boundary (parametrized by $\epsilon$ ) of five-dimensional anti-de Sitter space-time. The $2 \mathrm{~d}$ surface $\Sigma$ that separates two subsystems in the entanglement entropy calculation is, thus, defined on the regularized boundary. The parameter $\epsilon$ plays the role of the UV cut-off on the quantum field theory side. According to the proposal of Ryu and Takayanagi [18], [19] in the anti-de Sitter space-time one considers a minimal $3 \mathrm{~d}$ surface $\Gamma$ which bounds the surface $\Sigma$. The quantity

$$
S=\frac{\operatorname{Area}(\Gamma)}{4 G_{N}},
$$

where $G_{N}$ is five-dimensional Newton's constant, then is equal to entanglement entropy (3.3) in the boundary quantum conformal field theory. This proposal has been verified in many particular cases and there are reasons why it should be valid in general [21. In this section we use this holographic interpretation in order to determine the parameter $\mu$ in (3.2) and (2.11).

To simplify things, we consider the case of flat four-dimensional space-time. Then the logarithmic term in the entropy (3.2) or (4.1) is entirely due to the extrinsic curvature of $\Sigma$. We note that in flat space-time $R_{\Sigma}=k_{a} k_{a}-\operatorname{tr} k^{2}$, as follows from (A.5).

The result of the holographic calculation (4.1) can be presented in general form

$$
S=\frac{A(\Sigma)}{4 \pi \epsilon^{2}} N^{2}-\frac{N^{2}}{24 \pi} \int_{\Sigma}\left(\frac{\gamma}{2} k_{a} k_{a}+\beta \operatorname{tr} k^{2}\right) \ln \epsilon+. .,
$$

where ".." stands for the finite part and we use that in the holographic correspondence

$\frac{1}{G_{N}}=\frac{2 N^{2}}{\pi}$. We further consider two choices of surface $\Sigma$, a two-dimensional cylinder and 
sphere $S_{2}$, determine the constants $\gamma$ and $\beta$ and then compare (4.2) with (3.3), (3.2) to determine value of parameter $\mu$.

Generally, the holographic calculation of the logarithmic term in (3.3), (4.2) is related to the surface anomalies studied by Graham and Witten [30] (see also [31] and [32]).

Two-dimensional cylinder. We choose the AdS metric in the form (and fix AdS radius $l=1)$

$$
d s^{2}=\frac{d \rho^{2}}{4 \rho^{2}}+\frac{1}{\rho}\left(-d t^{2}+d z^{2}+d r^{2}+r^{2} d \phi^{2}\right)
$$

The surface $\Sigma$ corresponds to values of the coordinates $\rho=0, r=a, 0 \leq \phi \leq 2 \pi$, $0 \leq z \leq L$, where we introduced $L$ in order to regularize the length of the cylinder. The surface $\Gamma$ is described by function $r(\rho)$. It has area

$$
\operatorname{Area}(\Gamma)=2 \pi L \int_{\epsilon^{2}} \frac{d \rho}{2 \rho^{2}} r(\rho) \sqrt{1+4 \rho r^{\prime 2}}
$$

where $r^{\prime}=\partial_{\rho} r(\rho)$. The minimal surface is described by equation

$$
\sqrt{1+4 \rho r^{\prime 2}}=\rho^{2} \partial_{\rho}\left(\frac{4 r r^{\prime}}{\rho \sqrt{1+4 \rho r^{\prime 2}}}\right)
$$

For small $\rho$, the asymptotic solution of this equation that approaches $\Sigma$ at $\rho=0$ is

$$
r(\rho)=a-\frac{\rho}{4 a}+O\left(\rho^{2}\right)
$$

Respectively, the asymptotic behavior of the area is

$$
\operatorname{Area}(\Gamma)=\pi L a \int_{\epsilon^{2}} \frac{d \rho}{\rho^{2}}\left(1-\frac{1}{8 a^{2}} \rho+. .\right)=\pi L a\left(\frac{1}{\epsilon^{2}}+\frac{1}{4 a^{2}} \ln \epsilon+. .\right) .
$$

Although expression (4.7) is asymptotic the finite part in (4.7) can be easily restored. Indeed, the metric (4.3) is invariant under rescaling, $\rho \rightarrow \lambda^{2} \rho, t \rightarrow \lambda t, z \rightarrow \lambda z$ and $r \rightarrow \lambda r$. This invariance determines, up to an additive numerical constant, the finite part. Thus, the holographic entropy in the case of cylinder is

$$
S=\frac{A(\Sigma)}{4 \pi \epsilon^{2}} N^{2}+\frac{N^{2}}{8} \frac{L}{a}\left(\ln \frac{\epsilon}{a}+c_{1}\right)+c_{2}
$$

where $c_{1}$ and $c_{2}$ are some constants.

In order to compare this to (4.2) we have to know the extrinsic curvature of the cylinder. In flat space-time one of the normal vectors to surface $\Sigma$ is timelike, $n_{1}^{t}=1$. The corresponding extrinsic curvature vanishes. The other normal vector is $n_{2}^{r}=1$. It has the only non-vanishing component of the extrinsic curvature $k_{\phi \phi}^{2}=a$. Thus, one has that

$$
k_{a} k_{a}=\frac{1}{a^{2}}, \quad \operatorname{tr} k^{2}=\frac{1}{a^{2}}
$$

Comparing now (4.8) and (4.2) we find a relation

$$
\gamma+2 \beta=-3
$$


Sphere $S_{2}$. The AdS metric is

$$
d s^{2}=\frac{d \rho^{2}}{4 \rho^{2}}+\frac{1}{\rho}\left(-d t^{2}+d r^{2}+r^{2}\left(d \theta^{2}+\sin ^{2} \theta d \phi\right)\right) .
$$

The surface $\Sigma$ is defined by $\rho=0, r=a, 0 \leq \phi \leq 2 \pi$ and $0 \leq \theta \leq \pi$. The surface $\Gamma$ is described by function $r(\rho)$ and has area

$$
\operatorname{Area}(\Gamma)=4 \pi \int_{\epsilon^{2}} \frac{d \rho}{2 \rho^{2}} r^{2}(\rho) \sqrt{1+4 \rho r^{\prime 2}} .
$$

The minimal surface is described by equation

$$
r(\rho) \sqrt{1+4 \rho r^{\prime 2}}=\rho^{2} \partial_{\rho}\left(\frac{2 r^{2} r^{\prime}}{\sqrt{1+4 \rho r^{\prime 2}}}\right) .
$$

The asymptotic solution takes the form

$$
r(\rho)=a-\frac{1}{2 a} \rho+O(\rho)^{2} .
$$

The area of the minimal surface is

$$
\operatorname{Area}(\Gamma)=2 \pi a^{2} \int_{\epsilon^{2}} \frac{d \rho}{\rho^{2}}\left(1-\frac{1}{2 a^{2}} \rho+. .\right)=\frac{2 \pi a^{2}}{\epsilon^{2}}+2 \pi \ln \epsilon+. .
$$

The finite part in (4.15) is determined by the invariance of metric (4.11) under rescaling, $\rho \rightarrow \lambda \rho, t \rightarrow \lambda t, r \rightarrow \lambda r$. Applying the holographic proposal one finds that entanglement entropy in the case of sphere is (see also [19])

$$
S=\frac{A(\Sigma)}{4 \pi \epsilon^{2}} N^{2}+N^{2} \ln \frac{\epsilon}{a}+c_{2}^{\prime}
$$

Vector $n_{1}^{t}=1$ normal to $\Sigma$ has zero extrinsic curvature. The other vector normal to $\Sigma, n_{2}^{r}=1$, has the non-vanishing extrinsic curvature $k_{\theta \theta}^{2}=a, k_{\phi \phi}^{2}=a \sin ^{2} \theta$. Hence, one has that

$$
k_{a} k_{a}=\frac{4}{a^{2}}, \quad \operatorname{tr} k^{2}=\frac{2}{a^{2}} .
$$

By comparing (4.16) with (4.2) one finds that

$$
\gamma+\beta=-3
$$

Both relations, (4.10) and (4.18), are consistent if

$$
\gamma=-3, \quad \beta=0
$$

The comparison of (3.3), (3.2) with (4.2) for values (4.19) determines the value of the parameter $\mu$ in (3.2) and (2.11),

$$
\mu=-1
$$




\section{Generic 4d conformal field theory}

The result (1.1) for entanglement entropy is easily generalized for a generic conformal field theory in four dimensions. The conformal anomaly of such a theory is a combination of the type A and type B anomalies, the surface term taking the form (2.11) with value of parameter $\mu=-1$, already determined in section 4 by using the holographic correspondence. Entanglement entropy of the theory is then given by (2.7),

$$
S_{(A, B)}=\frac{A(\Sigma)}{4 \pi \epsilon^{2}}+\left(A a_{A}^{\Sigma}+B a_{B}^{\Sigma}\right) \ln \epsilon+s_{(A, B)}(g)
$$

where $a_{A}^{\Sigma}$ and $a_{B}^{\Sigma}$ are given by (2.11) (with $\mu=-1$ ). The contribution of the type A anomaly is always determined by topology of surface $\Sigma$ while the dependence of the entropy on the extrinsic geometry of $\Sigma$ is due to the type B anomaly.

In flat space-time only the extrinsic curvature contributes to $a_{2}^{\Sigma}$ and one has that

$$
S_{(A, B)}=\frac{A(\Sigma)}{4 \pi \epsilon^{2}}+\frac{\pi}{8}\left(A \int_{\Sigma} R_{\Sigma}+B \int_{\Sigma}\left(\operatorname{tr} k^{2}-\frac{1}{2} k_{a} k_{a}\right)\right) \ln \epsilon+s_{(A, B)}(g)
$$

where $R_{\Sigma}=k_{a} k_{a}-\operatorname{tr} k^{2}$ in flat space-time. The finite part $s_{(A, B)}$ in (5.2) can be determined, using the rescaling property of $s_{(A, B)}$, for some geometries, in particular for sphere $S_{2}$ and the two-dimensional cylinder. Using the expressions, obtained in section 4 , for the extrinsic curvature of these two surfaces in flat space-time one finds that the entanglement entropy of a generic CFT in the case of cylinder is

$$
S_{(A, B)}^{\text {cylinder }}=\frac{A(\Sigma)}{4 \pi \epsilon^{2}}+B \frac{\pi^{2}}{8} \frac{L}{a} \ln \frac{\epsilon}{a}
$$

and

$$
S_{(A, B)}^{\text {sphere }}=\frac{A(\Sigma)}{4 \pi \epsilon^{2}}+A \pi^{2} \ln \frac{\epsilon}{a}
$$

in the case of sphere $S_{2}$. Thus, these two geometries single out two different types of conformal anomaly in the calculation of entanglement entropy.

\section{Conclusions}

The dependence of entanglement entropy on the extrinsic geometry of the surface $\Sigma$ that separates two subsystems is a missing element in the previous study of entanglement entropy (see, however, 12 for an earlier discussion). In particular, the extrinsic geometry plays an important role in the entanglement entropy calculation in flat space-time. In this paper we use the conformal symmetry and the holographic correspondence and fully specify the way the extrinsic curvature appears in entanglement entropy. The case of the conformal field theory dual to a gravity on anti-de Sitter space-time is considered in detail. We extend this consideration to a generic conformal field theory in four dimensions. In particular, we obtain a closed-form expression for entanglement entropy of a generic CFT in the case when $\Sigma$ is a two-dimensional cylinder and when $\Sigma$ is two-dimensional sphere. We specify the way the type A and the type B conformal anomalies show up in the entanglement entropy. 


\section{Acknowledgments}

I thank D. Fursaev for helpful remarks and S. Theisen for email communication. 


\section{Appendix}

\section{A Conformal symmetry and the extrinsic geometry}

In this section we collect the useful formulas for the conformal transformation of various geometric quantities defined on a co-dimension 2 submanifold $\Sigma$.

Under the transformation $g_{\mu \nu} \rightarrow e^{-2 \omega} g_{\mu \nu}, n_{\mu}^{a} \rightarrow e^{-\omega} n_{\mu}^{a}, a=1,2$ the extrinsic curvature $k_{\mu \nu}^{a}=-\gamma_{\mu}^{\alpha} \gamma_{\nu}^{\beta} \nabla_{\alpha} n_{\beta}^{a}, k^{a}=\gamma^{\mu \nu} k_{\mu \nu}^{a}$ of $\Sigma$ changes as follows

$$
\begin{gathered}
k_{\mu \nu}^{a} \rightarrow e^{-\omega}\left(k_{\mu \nu}^{a}+\gamma_{\mu \nu} n_{a}^{\alpha} \nabla_{\alpha} \omega\right) \\
k_{a} \rightarrow e^{-\omega}\left(k_{a}+(d-2) n_{a}^{\alpha} \nabla_{\alpha} \omega\right)
\end{gathered} .
$$

The projections of the Riemann and Ricci tensors, $R_{a b a b}=R_{\alpha \beta \mu \nu} n_{a}^{\alpha} n_{b}^{\beta} n_{a}^{\mu} n_{b}^{\nu}, R_{a a}=$ $R_{\alpha \beta} n_{a}^{\alpha} n_{a}^{\beta}$, on the subspace orthogonal $\Sigma$ transform as

$$
\begin{aligned}
& R_{a b a b} \rightarrow e^{2 \omega}\left(R_{a b a b}+2 n_{a}^{\alpha} n_{b}^{\beta} \nabla_{\alpha} \nabla_{\beta} \omega+2\left(n_{a}^{\alpha} \partial_{\alpha} \omega\right)\left(n_{a}^{\alpha} \partial_{\alpha} \omega\right)-2(\nabla \omega)^{2}\right) \\
& R_{a a} \rightarrow e^{2 \omega}\left(R_{a a}+(d-2) n_{a}^{\alpha} n_{a}^{\beta}\left(\nabla_{\alpha} \nabla_{\beta} \omega+\nabla_{\alpha} \omega \nabla_{\beta} \omega\right)+2(2-d)(\nabla \omega)^{2}+2 \Delta \omega\right) .
\end{aligned}
$$

The Laplace operator $\Delta$ is further presented in the form

$$
\Delta \omega=\hat{\Delta} \omega+n_{a}^{\alpha} n_{a}^{\beta} \nabla_{\alpha} \nabla_{\beta} \omega-k_{a} n_{a}^{\alpha} \partial_{\alpha} \omega
$$

where $\hat{\Delta}$ is the intrinsic Laplacian on $\Sigma$.

In dimension $d=4$ there are two conformal covariant combinations of the Riemann curvature and the extrinsic curvature [25],

$$
\begin{aligned}
& \left(2 R_{a b a b}-R_{a a}-\frac{1}{2} k_{a} k_{a}\right) \rightarrow e^{2 \omega}\left(2 R_{a b a b}-R_{a a}-\frac{1}{2} k_{a} k_{a}-2 \hat{\Delta} \omega\right), \\
& \left(\operatorname{tr} k^{2}-\frac{1}{2} k_{a} k_{a}\right) \rightarrow e^{2 \omega}\left(\operatorname{tr} k^{2}-\frac{1}{2} k_{a} k_{a}\right) .
\end{aligned}
$$

Using the Gauss-Codazzi equation

$$
R=R_{\Sigma}+2 R_{a a}-R_{a b a b}-k_{a} k_{a}+\operatorname{tr} k^{2}
$$

where $R_{\Sigma}$ is the intrinsic Ricci scalar of the surface, one finds that the conformal covariant combination involving the Ricci scalar is

$$
\left(3 R_{a a}-2 R-\frac{3}{2} k_{a} k_{a}\right)=\left(2 R_{a b a b}-R_{a a}-\frac{1}{2} k_{a} k_{a}\right)-2 R_{\Sigma}+2\left(\frac{1}{2} k_{a} k_{a}-\operatorname{tr} k^{2}\right)
$$




\section{References}

[1] L. Bombelli, R. K. Koul, J. H. Lee and R. D. Sorkin, Phys. Rev. D 34, 373 (1986).

[2] M. Srednicki, Phys. Rev. Lett. 71, 666 (1993).

[3] V. P. Frolov and I. Novikov, Phys. Rev. D 48, 4545 (1993).

[4] C. G. Callan and F. Wilczek, Phys. Lett. B 333, 55 (1994).

[5] C. Holzhey, F. Larsen and F. Wilczek, Nucl. Phys. B 424, 443 (1994).

[6] S. N. Solodukhin, Phys. Rev. D 51, 609 (1995).

[7] S. N. Solodukhin, Phys. Rev. D 51, 618 (1995).

[8] D. V. Fursaev and S. N. Solodukhin, Phys. Lett. B 365, 51 (1996).

[9] D. V. Fursaev, Phys. Rev. D 51, 5352 (1995).

[10] S. N. Solodukhin, Phys. Rev. D 57, 2410 (1998).

[11] R. B. Mann and S. N. Solodukhin, Nucl. Phys. B 523, 293 (1998).

[12] R. B. Mann and S. N. Solodukhin, Phys. Rev. D 54, 3932 (1996).

[13] H. Casini and M. Huerta, Phys. Lett. B 600, 142 (2004).

[14] V. Korepin, Phys. Rev. Lett. 92, 096402 (2004).

[15] P. Calabrese and J. L. Cardy, J. Stat. Mech. 0406, P002 (2004).

[16] M. S. Berger and R. V. Buniy, "Entanglement Entropy and Spatial Geometry," arXiv:0801.4564 [hep-th].

[17] R. Brustein, M. B. Einhorn and A. Yarom, JHEP 0601, 098 (2006).

[18] S. Ryu and T. Takayanagi, Phys. Rev. Lett. 96, 181602 (2006).

[19] S. Ryu and T. Takayanagi, JHEP 0608, 045 (2006).

[20] R. Emparan, JHEP 0606, 012 (2006).

[21] D. V. Fursaev, JHEP 0609, 018 (2006).

[22] S. N. Solodukhin, Phys. Rev. Lett. 97, 201601 (2006).

[23] I. R. Klebanov, D. Kutasov and A. Murugan, "Entanglement as a Probe of Confinement," arXiv:0709.2140 [hep-th].

[24] V. E. Hubeny, M. Rangamani and T. Takayanagi, JHEP 0707, 062 (2007).

[25] J. S. Dowker, Phys. Rev. D 50, 6369 (1994).

[26] D. V. Fursaev and S. N. Solodukhin, Phys. Rev. D 52, 2133 (1995). 
[27] A. Schwimmer and S. Theisen, "Entanglement Entropy, Trace Anomalies and Holography," arXiv:0802.1017 [hep-th].

[28] D. V. Fursaev, Phys. Lett. B 334, 53 (1994).

[29] M. Henningson and K. Skenderis, JHEP 9807, 023 (1998).

[30] C. R. Graham and E. Witten, Nucl. Phys. B 546, 52 (1999).

[31] M. Henningson and K. Skenderis, JHEP 9906, 012 (1999).

[32] D. E. Berenstein, R. Corrado, W. Fischler and J. M. Maldacena, Phys. Rev. D 59, 105023 (1999).

[33] D. V. Vassilevich, Phys. Rept. 388, 279 (2003). 